\title{
New graviton mass bound from binary pulsars
}

\author{
Lijing Shao $\odot,{ }^{1,2,3,}{ }^{*}$ Norbert Wex $\odot,{ }^{2}$ and Shuang-Yong Zhou $\odot^{4,5}$ \\ ${ }^{1}$ Kavli Institute for Astronomy and Astrophysics, Peking University, Beijing 100871, China \\ ${ }^{2}$ Max-Planck-Institut für Radioastronomie, Auf dem Hügel 69, D-53121 Bonn, Germany \\ ${ }^{3}$ National Astronomical Observatories, Chinese Academy of Sciences, Beijing 100012, China \\ ${ }^{4}$ Interdisciplinary Center for Theoretical Study, University of Science and Technology of China, Hefei, Anhui 230026, China \\ ${ }^{5}$ Peng Huanwu Center for Fundamental Theory, Hefei, Anhui 230026, China
}

(Received 24 May 2020; accepted 8 July 2020; published 23 July 2020)

\begin{abstract}
In Einstein's general relativity, gravity is mediated by a massless metric field. The extension of general relativity to consistently include a mass for the graviton has profound implications for gravitation and cosmology. Salient features of various massive gravity theories can be captured by Galileon models, the simplest of which is the cubic Galileon. The presence of the Galileon field leads to additional gravitational radiation in binary pulsars where the Vainshtein mechanism is less suppressed than its fifth-force counterpart, which deserves a detailed confrontation with observations. We prudently choose 14 well-timed binary pulsars, and from their intrinsic orbital decay rates we put a new bound on the graviton mass, $m_{g} \lesssim 2 \times 10^{-28} \mathrm{eV} / c^{2}$ at the $95 \%$ confidence level, assuming a flat prior on $\ln m_{g}$. It is equivalent to a bound on the graviton Compton wavelength $\lambda_{g} \gtrsim 7 \times 10^{21} \mathrm{~m}$. Furthermore, we extensively simulate times of arrival for pulsars in orbit around stellar-mass black holes and the supermassive black hole at the Galactic Center, and we investigate their prospects of probing the cubic Galileon theory in the near future.
\end{abstract}

DOI: $10.1103 /$ PhysRevD.102.024069

\section{INTRODUCTION}

The late-time accelerated cosmic expansion poses a profound challenge for modern physics that is known as the dark energy problem [1-3]. From observations, we know that dark energy manifests at length scales significantly larger than the galactic size or, in field-theoretic terminology, in the infrared regime. Dark energy is often hypothesized as a cosmological constant in the standard $\Lambda$ cold dark matter model [4], but its real nature remains elusive. Astrophysical objects (in particular, the type Ia supernovae) in the relatively nearby Universe and the cosmic microwave background in the early Universe provide two classes of independent probes to measure the Hubble expansion parameter of today, $H_{0}$. Recent observations from them, however, have inferred inconsistent values of $H_{0}$ at a significance level of $4.4 \sigma$ [5-7]. The discrepancy aggravates the dark energy puzzle and, in the meantime, has triggered tremendous interest in searching for new physics beyond the standard paradigm.

\footnotetext{
1shao@pku.edu.cn
}

Published by the American Physical Society under the terms of the Creative Commons Attribution 4.0 International license. Further distribution of this work must maintain attribution to the author(s) and the published article's title, journal citation, and DOI. Open access publication funded by the Max Planck Society.
One of the main approaches to explaining dark energy phenomena involves infrared modifications to the canonical gravity theory, general relativity (GR) [8-10]. Modifications usually introduce extra field contents, with a scalar degree of freedom being the simplest and the most widely investigated in the literature. However, such a new scalar is likely to bring in a fifth force [8-10], which is stringently constrained by observations in the Solar System [11] and binary pulsars [12-17]. Therefore, to successfully account for the accelerated expansion of the Universe, we need a modified gravity theory where the theory gives rise to order-1 corrections at cosmological scales but deviations from GR are extremely suppressed in the Solar System, which makes nonlinearity a crucial ingredient in the theory. For a class of such infrared modifications of gravity, including the Galileon models $[18,19]$, this is achieved by the Vainshtein mechanism [20,21], by which the new scalar becomes nonlinearly coupled in the local dense environment, thus suppressing the fifth force in the Solar System. The length scale within which the scalar becomes strongly coupled is called the Vainshtein radius, $r_{\star}$, and it is only outside of the Vainshtein radius that the linear perturbations can be trusted. It is important that when dealing with models with the Vainshtein mechanism the full nonlinear theory, as opposed to the linear theory, needs to be solved to make the correct physical interpretations.

The Vainshtein mechanism is intimately related to massive gravity, and the accelerated cosmic expansion 
may be due to a condensate of gravitons with a Hubblescale mass $[22,23]$. It was pointed out in the 1970s that the unique (Lorentz invariant) linear theory of massive gravity deviates from GR by order-1 corrections, known as the van Dam-Veltman-Zakharov discontinuity [24-26]. Vainshtein soon thereafter suggested that this cannot be used to rule out massive gravity and that instead one needs to solve the nonlinear theory in environments such as the Solar System to get the right prediction [20]. In other words, while the conventional helicity- 2 modes of GR become strongly coupled at the Schwarzschild radius, the extra modes of massive gravity becomes strongly coupled within a much larger Vainshtein radius for the same central mass. To extract the most strongly coupled extra modes in massive gravity, one takes the decoupling limit per the de RhamGabadadze-Tolley (dRGT) tuning [27,28],

$m_{g} \rightarrow 0, \quad M_{\mathrm{Pl}} \rightarrow \infty, \quad \Lambda=\left(m_{g}^{2} M_{\mathrm{Pl}}\right)^{1 / 3} \rightarrow$ fixed

where $m_{g}$ is the graviton mass and $M_{\mathrm{Pl}} \equiv 1 / \sqrt{8 \pi G}$ is the reduced Planck mass, and obtains a scalar effective field theory with the Galileon symmetry

$$
\pi_{s} \rightarrow \pi_{s}+a+b_{\mu} x^{\mu},
$$

where $\pi_{s}$ is the Galileon field and $a$ and $b_{\mu}$ are constants. The decoupling limit (1) scales away the small effects due to other modes and lets us focus on the physics most important near the scale of $\Lambda$. In the presence of matter sources, we also take the limit where the energymomentum tensor $T_{\mu \nu}$ goes to infinity but the ratio between $T_{\mu \nu} / M_{\mathrm{Pl}}$ is fixed. The Galileon scalar then couples to the trace of $T_{\mu \nu}$ and encapsulates the salient features, including the Vainshtein mechanism, of the extra modes in massive gravity $[18,19]$.

In this paper, we study the simplest model that exhibits the Vainshtein mechanism-namely, the cubic Galileon [18]. This model is the decoupling limit of the DvaliGabadadze-Porrati braneworld model [18,29], where the graviton acquires a so-called soft mass from embedding the 3-brane Universe in a four-dimensional bulk with an Einstein-Hilbert term. The Galileon models are also the decoupling limit of the recently discovered dRGT model [27,28], a unique nonlinear (Lorentz invariant) massive gravity with a so-called hard mass. The bigravity [30] or multigravity extension of the dRGT model also leads to a bi-Galileon or multi-Galileon theory [31-33]. Therefore, the cubic Galileon model is often taken as a proxy to cover all of the Lorentz invariant massive gravity models, though by no means does it encode all aspects of a complete theory of massive gravity where other terms, for example, the quartic Galileon term, might appear.

Horndeski theory [34], the generalized scalar-tensor theory with up to second derivatives in field equations, can be rederived in the Galileon framework [35]. It is worth mentioning that, while a large class of Horndeski models have been ruled out by the coincident observation of the gravitational-wave signal [36] and the electromagnetic counterpart [37] from a binary neutron star inspiral GW170817 [38-40], ${ }^{1}$ the cubic Galileon subset of Horndeski theory, though being a simple straw man model, is still comfortably alive. Consequently, it is intriguing to study the cubic Galileon in light of recent research activities in the field.

The fifth-force effects of massive gravity are often screened by the Vainshtein mechanism so effectively that the existing constraints in the dense environment can easily be evaded [42], and only at a cosmological density in the infrared regime can the theory deviate significantly from GR, accounting for the dark energy. However, de Rham et al. [43] found that, in binary pulsar systems, the suppression factor in the extra gravitational radiation due to the Galileon mode is less than the suppression factor in the static fifth-force effect (see Sec. II). Therefore, it becomes extremely interesting to check with the existing tests related to the gravitational radiation in binary pulsars for the cubic Galileon model.

In this work, we present a thorough phenomenological study of the Galileon radiation for binary pulsar systems. We carefully choose 14 well-timed binary pulsars to put constraints on the theory parameter of the cubic Galileon. Among these pulsars, recent observations of the double pulsar PSR J0737-3039A [44,45] give the strongest bound on the graviton mass, $m_{g} \lesssim 3 \times 10^{-28} \mathrm{eV} / c^{2}$ at the $95 \%$ confidence level (C.L.). Furthermore, a combination of all 14 pulsars in the Bayesian framework gives

$$
m_{g} \lesssim 2 \times 10^{-28} \mathrm{eV} / c^{2} \quad(95 \% \text { C.L. }),
$$

with a flat prior on $\ln m_{g}$. It translates into a limit on the graviton Compton wavelength $\lambda_{g} \gtrsim 7 \times 10^{21} \mathrm{~m}$.

The paper is organized as follows. In Sec. II, we review the basics for the Galileon radiation [43]. In Sec. III, systematic studies are carried out to understand the dependence of the Galileon radiation on system parameters and the figure of merit to test it. Based on these studies, we choose 14 binary pulsars to cast tight constraints on the graviton mass. Limits are obtained from individual pulsars as well as a combination of them. Moreover, in Sec. IV, with a set of simulated times of arrival for near-future radio telescopes, we investigate the prospects for using pulsars around a stellar-mass black hole (BH) companion [46-48] and the supermassive $\mathrm{BH}$ at the Galactic Center (namely, Sgr A*) [49-52] to constrain the cubic Galileon theory. Section V presents a discussion and briefly summarizes the paper.

\footnotetext{
${ }^{1}$ Care, however, must be taken to interpret this result, as the observed gravitational-wave frequencies are very close to the cutoff of the Horndeski theory as an effective field theory [41].
} 
Throughout the paper, we implicitly assume units where $\hbar=c=1$, except for a couple of places where $\hbar$ and $c$ are restored for the reader's convenience.

\section{THEORY}

Since the Hulse-Taylor pulsar provided the first indirect evidence for the existence of gravitational waves [53], many more binary pulsars have been playing an important role in probing the properties of the gravitational radiation in alternative gravity theories [12,45,54-57]. We briefly review the gravitational radiation for a binary pulsar in GR and in cubic Galileon theory in Secs. II A and II B, respectively.

\section{A. General relativity}

Consider a binary system with component masses $m_{1}$ and $m_{2}$ in an orbit with a semimajor axis $a$ and an eccentricity $e$. Because of the finite propagating velocity of gravity, at leading order the binary loses energy by radiating off gravitational waves with an emitting power [58]

$\mathcal{P}_{\mathrm{GR}}=\frac{32 \eta^{2}}{5 c^{5}} \frac{G^{4} M^{5}}{a^{5}}\left(1+\frac{73}{24} e^{2}+\frac{37}{96} e^{4}\right)\left(1-e^{2}\right)^{-7 / 2}$,

where the total mass $M \equiv m_{1}+m_{2}$, the symmetric mass ratio $\eta \equiv m_{1} m_{2} / M^{2}$, and $G$ and $c$ are the gravitational constant and the speed of light, respectively.

From Kepler's third law for a binary system, we have

$$
n_{b}^{2} a^{3}=G M,
$$

where $n_{b} \equiv 2 \pi / P_{b}$, with $P_{b}$ the orbital period. The nonrelativistic orbital energy at the Newtonian order for the binary reads

$$
E_{b}=-\frac{\eta G M^{2}}{2 a} .
$$

Taking the time derivatives in Eqs. (5) and (6), we have

$$
\frac{\dot{a}}{a}=\frac{2}{3} \frac{\dot{P}_{b}}{P_{b}}
$$

and

$$
\frac{\dot{E}_{b}}{E_{b}}=-\frac{\dot{a}}{a}
$$

Finally, using the energy conservation law in GR, $\mathcal{P}_{\mathrm{GR}}=-\dot{E}_{b}$, we have [58]

$$
\begin{aligned}
\dot{P}_{b}^{\mathrm{GR}}= & -\frac{192 \pi}{5 c^{5}} \eta(G M)^{5 / 3} n_{b}^{5 / 3}\left(1+\frac{73}{24} e^{2}+\frac{37}{96} e^{4}\right) \\
& \times\left(1-e^{2}\right)^{-7 / 2} .
\end{aligned}
$$

\section{B. Cubic Galileon}

As discussed in the Introduction, (Lorentz invariant) massive gravity models in the decoupling limit essentially reduce to Galileon models plus linearized helicity-2 modes. For the cubic Galileon, we focus on the action [18,43]

$$
\begin{aligned}
S= & \int \mathrm{d}^{4} x\left[-\frac{1}{4} h^{\mu \nu}(\mathcal{E} h)_{\mu \nu}+\frac{h^{\mu \nu} T_{\mu \nu}}{2 M_{\mathrm{Pl}}}\right. \\
& \left.-\frac{3}{4}\left(\partial \pi_{s}\right)^{2}\left(1+\frac{1}{3 \Lambda^{3}} \square \pi_{s}\right)+\frac{\pi_{s} T}{2 M_{\mathrm{Pl}}}\right],
\end{aligned}
$$

where $h_{\mu \nu} \equiv g_{\mu \nu}-\eta_{\mu \nu}$ is the perturbation of the metric, the first two terms in the integrand are the linearized EinsteinHilbert term coupled to matter with the Lichnerowicz operator $(\mathcal{E} h)_{\mu \nu} \equiv-\frac{1}{2} \square h_{\mu \nu}+\cdots, T$ is the trace of the energy-momentum tensor $T^{\mu \nu}$, and $\Lambda$ is the strong coupling scale of the Galileon sector, related to the mass of graviton $m_{g}$ via $\Lambda^{3}=m_{g}^{2} M_{\mathrm{Pl}}$. Therefore, the field equations for $\pi_{s}$ and $h_{\mu \nu}$ decouple,

$$
\begin{gathered}
\frac{1}{M_{\mathrm{Pl}}} T_{\mu \nu}=-\frac{1}{2} \square h_{\mu \nu}, \\
\frac{1}{2 M_{\mathrm{Pl}}} T=\partial_{\mu}\left[-\frac{3}{2} \partial^{\mu} \pi_{s}\left(1+\frac{1}{3 \Lambda^{3}} \square \pi_{s}\right)+\frac{1}{4 \Lambda^{3}} \partial^{\mu}\left(\partial \pi_{s}\right)^{2}\right] .
\end{gathered}
$$

For a static system whose total mass is $M$, one can define the Vainshtein radius as

$$
r_{\star}=\left(\frac{M}{16 m_{g}^{2} M_{\mathrm{Pl}}^{2}}\right)^{1 / 3}=\frac{1}{\Lambda}\left(\frac{M}{16 M_{\mathrm{Pl}}}\right)^{1 / 3} .
$$

Within $r_{\star}$, the fifth force from the scalar degree of freedom is strongly suppressed.

In a static system, the fifth force is suppressed by a factor $\sim\left(L / r_{\star}\right)^{3 / 2}$, where $L$ is the typical length scale of the system [21]. For example, for an imaginary "static" binary system, we can choose it to be the semimajor axis of the orbit, $L \sim a$. However, binaries are not static. de Rham et al. [43] and Chu and Trodden [59] explicitly worked out the gravitational radiation behaviors in a time-dependent binary system at lowest orders. They found that (i) extra Galileon radiation powers exist at the monopole, dipole, and quadrupole levels, and (ii) for the dominant radiation the suppression factor is weakened from $\left(L / r_{\star}\right)^{3 / 2}$ to $\left(n_{b} r_{\star}\right)^{-3 / 2}$.

As with GR, the Newtonian-order contribution of both the monopole and dipole Galileon radiation vanishes, due to the conservation of energy and linear momentum of the system, respectively. Post-Newtonian-order contributions do exist for them. For the quadrupole radiation, the Newtonian-order contribution is nonzero. We collect the 
radiation powers of these multiple moments from Ref. [43] (see also the numerical calculation in Ref. [60]) in the following for later use in this work.

(a) Monopole radiation. In the cubic Galileon model (10), the monopole radiation power at leading order for a binary system is

$$
\mathcal{P}_{\text {mono }}=\frac{25}{48} \pi \beta^{2} n_{b}^{2} \frac{\left(n_{b} a\right)^{4}}{\left(n_{b} r_{\star}\right)^{3 / 2}} \frac{M_{\text {mono }}^{2}}{M_{\mathrm{Pl}}^{2}} \sum_{n=0}^{\infty}\left|I_{n}^{\text {mono }}(e)\right|^{2},
$$

where the constant $\beta$ and the "monopole mass" $M_{\text {mono }}$ (also known as the reduced mass) are defined, respectively, as
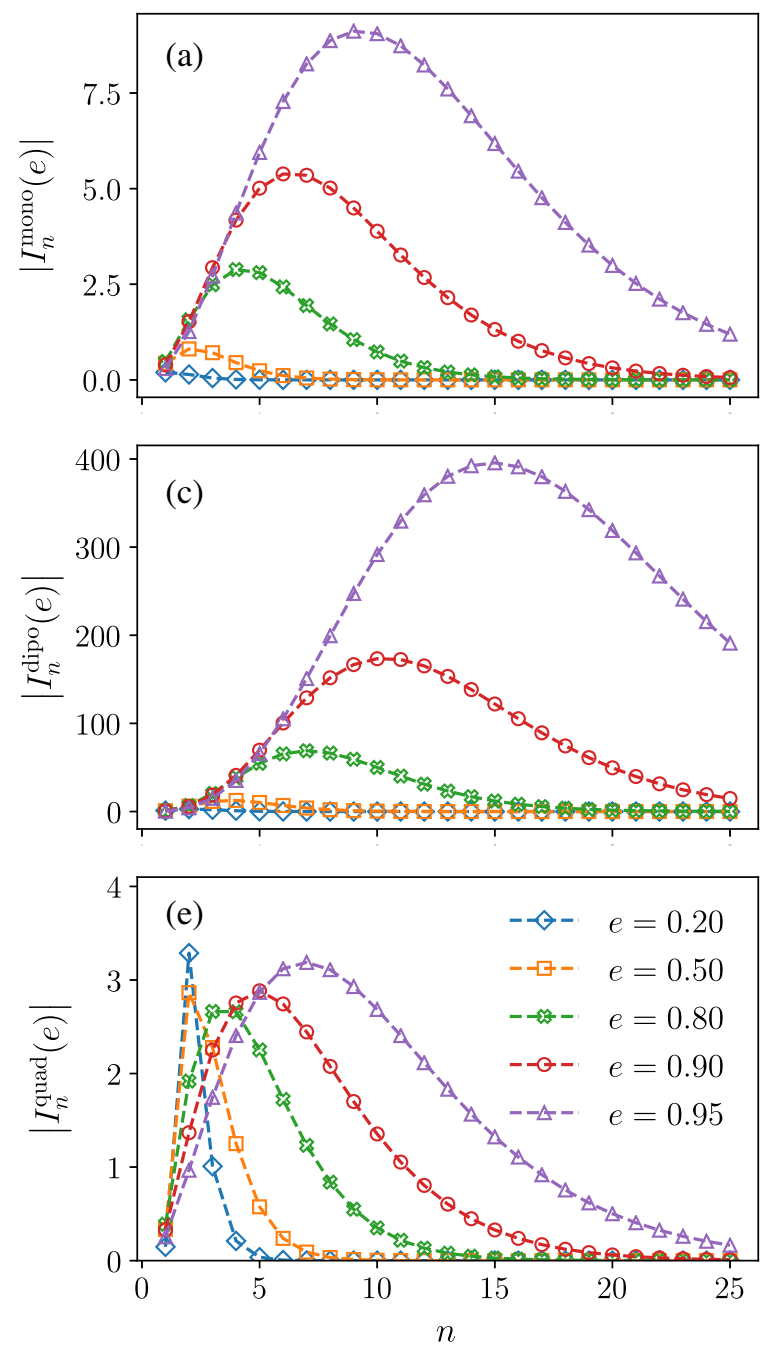

$$
\begin{gathered}
\beta \equiv \frac{3^{3 / 8}}{\Gamma(3 / 4)}\left(\frac{\pi}{32}\right)^{1 / 4} \simeq 0.6897, \\
M_{\text {mono }} \equiv \eta M=\frac{m_{1} m_{2}}{M},
\end{gathered}
$$

with $\Gamma(\cdot)$ the gamma function and the eccentricity mode function $I_{n}^{\text {mono }}(e)$ is as given in Eq. (24) and Fig. 1.

(b) Dipole radiation. The dipole radiation power at leading order for a binary system is

$$
\mathcal{P}_{\text {dipole }}=\frac{c_{1}^{2}}{8} n_{b}^{2} \frac{\left(n_{b} a\right)^{6}}{\left(n_{b} r_{\star}\right)^{3 / 2}} \frac{M_{\text {dipo }}^{2}}{M_{\mathrm{Pl}}^{2}} \sum_{n=0}^{\infty}\left|I_{n}^{\mathrm{dipo}}(e)\right|^{2},
$$
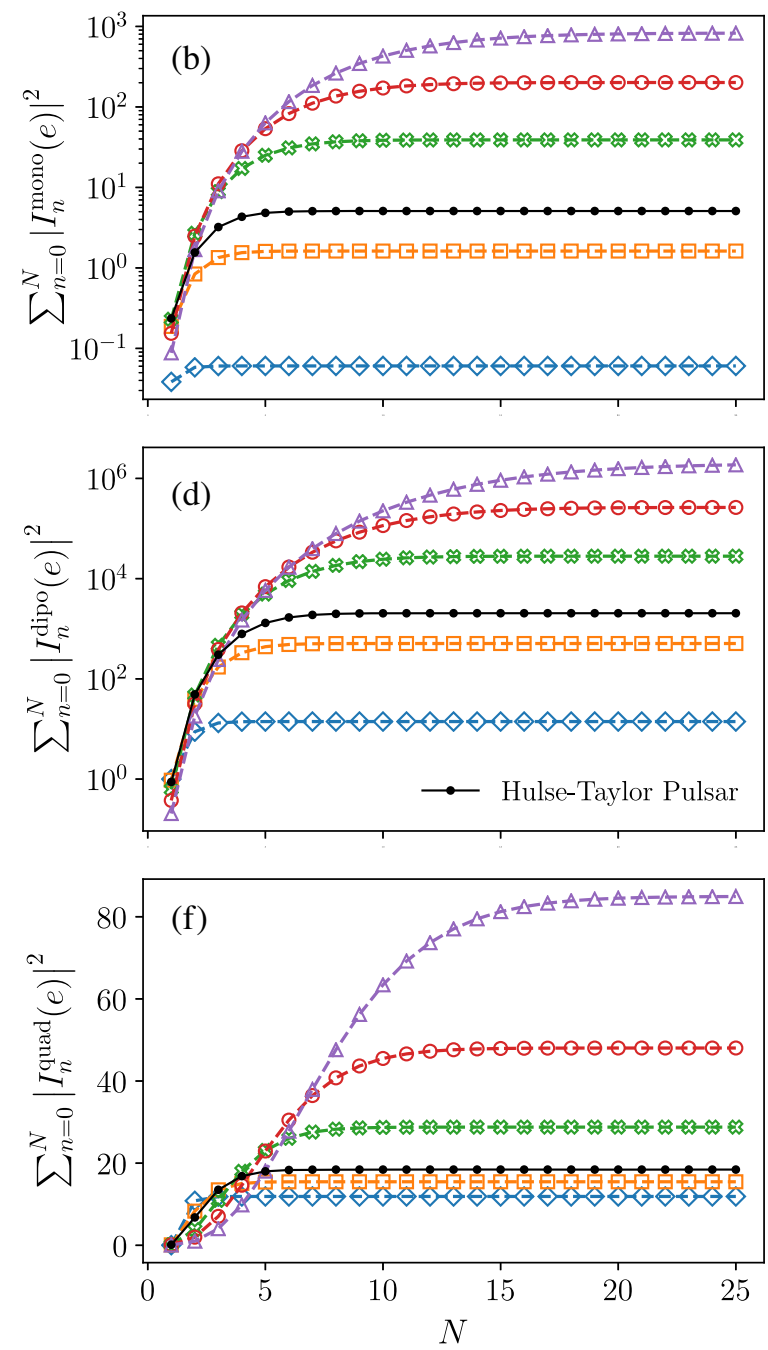

FIG. 1. Eccentricity mode functions for the Galileon radiation. (Left panels) Absolute values of $I_{n}^{\operatorname{mono}}(e), I_{n}^{\mathrm{dipo}}(e)$, and $I_{n}^{\text {quad }}(e)$ given from top to bottom. (Right panels) Cumulative contribution of these functions to the Galileon radiation power, in the form of (top panel) $\sum_{n=0}^{N}\left|I_{n}^{\text {mono }}(e)\right|^{2}$ for the monopole, (middle panel) $\sum_{n=0}^{N}\left|I_{n}^{\text {dipo }}(e)\right|^{2}$ for the dipole, and (bottom panel) $\sum_{n=0}^{N}\left|I_{n}^{\text {quad }}(e)\right|^{2}$ for the quadrupole. Notice that, for clarity, (b) and (d) use a logarithmic scale for the vertical axes, while all of the other axes use a linear scale. For a direct check, the solid black lines in the right column of panels reproduce the result in Fig. 1 of de Rham et al. [43] for the HulseTaylor pulsar PSR B1913 + 16, whose orbital eccentricity is $e \simeq 0.617$ [61]. 
where the constant $c_{1}$ and the "dipole mass" are defined, respectively, as

$$
\begin{array}{r}
c_{1} \equiv \frac{3^{7 / 8}(\pi / 2)^{1 / 4}}{8 \Gamma(7 / 4)}\left[1+\frac{3}{16} \frac{\Gamma(7 / 4)}{\Gamma(11 / 4)}\right] \simeq 0.4408, \\
M_{\text {dipo }} \equiv \mathcal{X} M_{\text {mono }}=M_{\text {mono }}\left(\frac{m_{1}-m_{2}}{M}\right),
\end{array}
$$

where $\mathcal{X} \equiv\left(m_{1}-m_{2}\right) / M$ and the eccentricity mode function $I_{n}^{\text {dipo }}(e)$ is as given in Eq. (25) and Fig. 1.

(c) Quadrupole radiation. Supplementing the quadrupole radiation power in Eq. (4), the extra power at leading order reads

$\mathcal{P}_{\text {quad }}=\frac{5 \lambda^{2}}{32} n_{b}^{2} \frac{\left(n_{b} a\right)^{3}}{\left(n_{b} r_{\star}\right)^{3 / 2}} \frac{M_{\text {quad }}^{2}}{M_{\mathrm{Pl}}^{2}} \sum_{n=0}^{\infty}\left|I_{n}^{\text {quad }}(e)\right|^{2}$,

where the constant $\lambda$ and the "quadrupole mass" $M_{\text {quad }}$ are defined, respectively, as

$$
\begin{gathered}
\lambda \equiv \frac{3^{9 / 8} \pi^{1 / 4}}{2^{17 / 4} \Gamma(9 / 4)} \simeq 0.2125, \\
M_{\text {quad }} \equiv \mathcal{Y} M_{\text {mono }}=M_{\text {mono }}\left(\frac{\sqrt{m_{1}}+\sqrt{m_{2}}}{\sqrt{M}}\right),
\end{gathered}
$$

where $\mathcal{Y} \equiv\left(\sqrt{m_{1}}+\sqrt{m_{2}}\right) / \sqrt{M}$ and the eccentricity mode function $I_{n}^{\text {quad }}(e)$ is as given in Eq. (26) and Fig. 1.

The eccentricity mode functions, $I_{n}^{\text {mono }}(e), I_{n}^{\text {dipo }}(e)$, and $I_{n}^{\text {quad }}(e)$, can be defined in a uniform way via the master function,

$$
I_{n}^{(p, q)}(e) \equiv \frac{n^{p+\frac{1}{4}}}{2 \pi}\left(1-e^{2}\right)^{p} \int_{0}^{2 \pi} \frac{e^{-i q x}}{(1+e \cos x)^{p}} \mathrm{~d} x .
$$

The above-mentioned eccentricity functions for monopole, dipole, and quadrupole radiations are

$$
\begin{gathered}
I_{n}^{\mathrm{mono}}(e)=I_{n}^{(p=2, q=n)}(e), \\
I_{n}^{\mathrm{dipo}}(e)=I_{n}^{(p=3, q=n-1)}(e), \\
I_{n}^{\text {quad }}(e)=I_{n}^{(p=3 / 2, q=n-2)}(e) .
\end{gathered}
$$

The behaviors of these functions are illustrated in Fig. 1 for different values of the eccentricity.

Using the same reasoning of energy balance in Sec. II A, we can get the extra contributions to $\dot{P}_{b}$ from the extra Galileon radiation powers in Eqs. (14), (17), and (20),

$$
\begin{aligned}
& \dot{P}_{b}^{\text {mono }}=-25 \sqrt{2} \beta^{2} \pi^{5 / 2} \frac{\eta(G M)^{7 / 6} n_{b}^{1 / 6}}{\hbar c^{3 / 2}} m_{g} \sum_{n=0}^{\infty}\left|I_{n}^{\text {mono }}(e)\right|^{2}, \\
& \dot{P}_{b}^{\text {dipo }}=-6 \sqrt{2} c_{1}^{2} \pi^{3 / 2} \frac{\eta \mathcal{X}^{2}(G M)^{11 / 6} n_{b}^{5 / 6}}{\hbar c^{7 / 2}} m_{g} \sum_{n=0}^{\infty}\left|I_{n}^{\text {dipo }}(e)\right|^{2},
\end{aligned}
$$

$\dot{P}_{b}^{\text {quad }}=-\frac{15}{\sqrt{2}} \lambda^{2} \pi^{3 / 2} \frac{\eta \mathcal{Y}^{2}(G M)^{5 / 6}}{n_{b}^{1 / 6} \hbar c^{1 / 2}} m_{g} \sum_{n=0}^{\infty}\left|I_{n}^{\text {quad }}(e)\right|^{2}$.

In obtaining these results, at leading order we used the Newtonian binding energy for the orbit in Eq. (6), and we made use of the orbital-averaged radiating powers as discussed above. Such a simplification is sufficient for the analysis in this paper. Notice that the extra contributions to $\dot{P}_{b}$ are all proportional to the graviton mass $m_{g}$, while in the linearized Fierz-Pauli theory $[48,62,63]$ the extra $\dot{P}_{b} \propto m_{g}^{2}$.

\section{CONSTRAINTS FROM BINARY PULSARS}

Now we would like to better understand the physical effect of the Galileon radiation in Eqs. (27)-(29) to different kinds of binary pulsars. We present some general considerations in Sec. III A concerning the dependence on the orbital eccentricity $e$, the orbital period $P_{b}$, and the component masses $\left(m_{1}, m_{2}\right)$. We cast constraints on the graviton mass in the cubic Galileon theory from precision timing of 14 carefully chosen binary pulsars in Sec. III B.

\section{A. General considerations}

From Fig. 1 we see that (i) given an eccentricity, the absolute values of the eccentricity mode functions, $I_{n}(e)$, increase at first, then have a peak at a particular $n=n^{\text {peak }}$ before they decrease; (ii) with the eccentricity increasing, $n^{\text {peak }}$ happens at a larger $n$-therefore, when we deal with highly eccentric binary pulsars, more modes are to be included in order to guarantee the convergence of the sum; and (iii) the cumulative contributions of these mode functions to the Galileon radiation powers in Eqs. (14), (17), and (20) saturate after a particular $n$, and for $e \lesssim 0.95$, summing up to $N \sim 15$ is generally sufficient. In the following calculation, we choose $N=30$ for better accuracy. However, one should remember that, for extremely eccentric binaries with $1-e \ll 10^{-2}$, a larger cutoff $N$ is needed.

Based on the contribution to the Galileon radiation power, we define

$$
f^{\mathrm{GR}}(e) \equiv\left(1+\frac{73}{24} e^{2}+\frac{37}{96} e^{4}\right)\left(1-e^{2}\right)^{-7 / 2}
$$




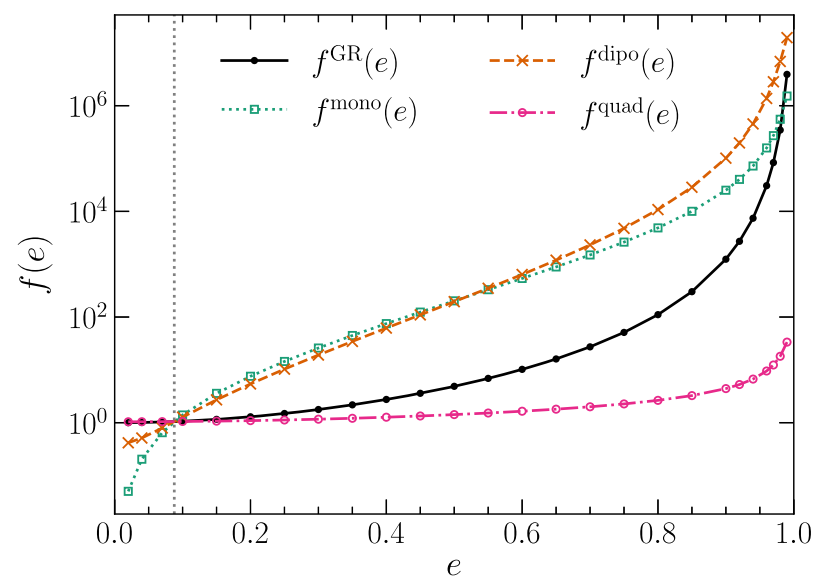

FIG. 2. The $f(e)$ factor in $\dot{P}_{b}$, defined in Eqs. (30)-(33), with a normalization such that $f^{\mathrm{GR}}=f^{\text {mono }}=f^{\text {dipo }}=f^{\text {quad }}$ for the double pulsar [44] whose $e \simeq 0.088$ (dotted vertical line).

and

$$
\begin{aligned}
f^{\text {mono }}(e) & \propto \sum_{n=0}^{\infty}\left|I_{n}^{\text {mono }}(e)\right|^{2}, \\
f^{\text {dipo }}(e) & \propto \sum_{n=0}^{\infty}\left|I_{n}^{\text {dipo }}(e)\right|^{2}, \\
f^{\text {quad }}(e) & \propto \sum_{n=0}^{\infty}\left|I_{n}^{\text {quad }}(e)\right|^{2} .
\end{aligned}
$$

These are the dependence shown in the GR radiation [Eq. (9)] and Galileon radiation [Eqs. (27)-(29)]. The proportional factors in Eqs. (31)-(33) can be arbitrary normalization values for the convenience of specific demonstration.

The $f(e)$ functions in Eqs. (30)-(33) are plotted in Fig. 2 with a choice of normalization. We can see that the more eccentric binaries are emitting more gravitational waves and contributing more significantly to $\dot{P}_{b}$. This is true for the three types of Galileon radiation, as well as the quadrupole radiation in GR. If the binary is extremely eccentric with $1-e \ll 1$, a huge amplification factor occurs, especially for the dipole radiation [Eq. (28)] in the cubic Galileon. As we will see below, the quadrupole Galileon radiation is, in general, the main contributor to $\dot{P}_{b}$ for binaries with comparable component masses [43]. The increase of $f^{\text {quad }}(e)$ at large eccentricities is slower than the other ones. Notice that the curves in the figure represent only one numerical factor in the radiation power, defined in Eqs. (30)-(33), while the other dependences (for example, the dependence on the orbital period and the component masses) are omitted here and will be investigated in the following. It is worth noting that the increased radiation due to eccentricity will generally tend to circularize the orbits (see, e.g., in GR [58]), making highly eccentric binaries less likely to exist at this point.

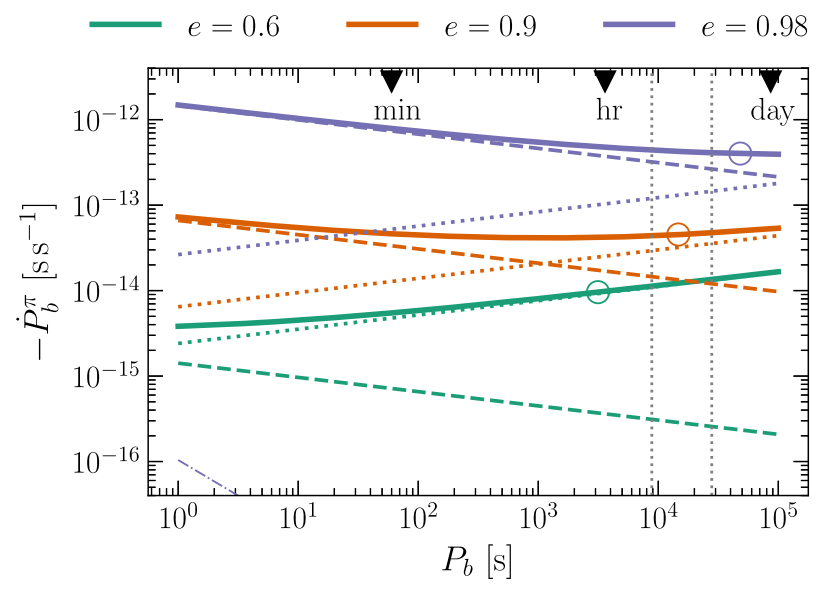

FIG. 3. Galileon radiation induced orbital decay as a function of the orbital period $P_{b}$ for three different eccentricities. We have assumed the graviton mass $m_{g}=10^{-27} \mathrm{eV} / c^{2}$ and component masses per the Hulse-Taylor pulsar-namely, $m_{1}=1.438 M_{\odot}$ and $m_{2}=1.390 M_{\odot}$. The dotted vertical lines indicate the orbital periods of (left) the double pulsar and (right) the Hulse-Taylor pulsar. The dashed, dot-dashed, and dotted lines indicate the contributions from monopole, dipole, and quadrupole radiation, respectively, while the solid lines are their sum. The dipole radiation is visible only for $e=0.98$ at the bottom left corner of the figure. Open circles on the solid curves represent systems that have lifetimes of $10^{6} \mathrm{yr}$ before merger in GR.

It is interesting to observe that, compared with $\dot{P}_{b}^{\mathrm{GR}} /$ $P_{b} \propto n_{b}^{8 / 3}$ in GR [see Eq. (9)], Galileon radiations have $\dot{P}_{b}^{\text {mono }} / P_{b} \propto n_{b}^{7 / 6}$ [see Eq. (27)], $\dot{P}_{b}^{\text {dipo }} / P_{b} \propto n_{b}^{11 / 6}$ [see Eq. (28)], and $\dot{P}_{b}^{\text {quad }} / P_{b} \propto n_{b}^{5 / 6}$ [see Eq. (29)]. It is a noteworthy feature for the gravitational radiation with the screening mechanism. With such a dependence, while more relativistic binaries are more prominent in emitting gravitational waves in GR, this may not always be true in the cubic Galileon. It resembles the dependence on $n_{b}$ in Lorentz-violating massive gravity [62,63] and gravity theories with a time-varying gravitational "constant" $G(t)[12,48]$.

In Fig. 3, we plot three types of Galileon radiation as a function of the orbital period for a binary pulsar system with component masses similar to the Hulse-Taylor pulsar, and a graviton mass $m_{g}=10^{-27} \mathrm{eV} / c^{2}$. First, we observe that the dipole radiation is orders of magnitude smaller than the other two types of Galileon radiation; thus the dipole radiation can be totally ignored. Though, as we recall from Fig. 2, the dependence of the $f(e)$ factor for the dipole radiation on the eccentricity $e$ is one of the steepest, the overall effect from dipole radiation is negligible even for $e=0.98$. Then we observe in Fig. 3 that, while for binaries with $e \lesssim 0.6$ the quadrupole radiation dominates [43], for highly eccentric binaries with relativistic orbits (namely, smaller $P_{b}$ ), the monopole radiation might dominate instead. This happens when $P_{b} \lesssim 10^{3} \mathrm{~s}$ for $e=0.9$, and $P_{b} \lesssim 10^{5}$ s for $e=0.98$. It is caused by the fact that $\dot{P}_{b}^{\text {mono }}$ 


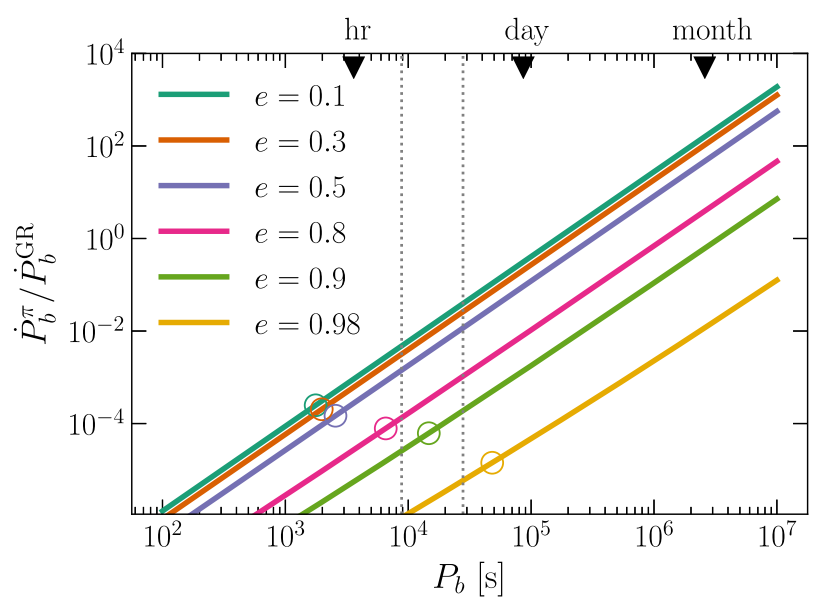

FIG. 4. The contribution from the Galileon radiation (summation of the monopole, dipole, and quadrupole radiations), relative to the quadrupole radiation in GR, for several different eccentricities. We have assumed $m_{1}, m_{2}$, and $m_{g}$ per Fig. 3 . The dotted vertical lines indicate the orbital periods for (left) the double pulsar and (right) the Hulse-Taylor pulsar. Open circles represent systems that have a lifetime of $10^{6} \mathrm{yr}$ before merger in GR. increases for larger $n_{b}$ (smaller $P_{b}$ ), while $\dot{P}_{b}^{\text {quad }}$ increases for smaller $n_{b}$ (larger $P_{b}$ ). Therefore, particularly for highly eccentric binaries to be discovered in the future, we should be cautious when considering only the quadrupole Galileon radiation. This also applies to highly eccentric galactic binaries (if they exist) in the millihertz gravitational-wave band for the Laser Interferometer Space Antenna (LISA) [64,65]. A similar discussion will also be mentioned for pulsar-BH systems in Sec. IV. In the following calculation, we keep all three types of Galileon radiation summed, regardless of their relative strength.

In Fig. 4, we plot the relative contribution of the Galileon radiation to the quadrupole radiation in GR. First, we see that the relative contribution increases when the orbital period is larger. This is in accordance with the screening mechanism, which works less well when the size increases [21], while the GR effects are more prominent when the orbit is more compact. Specifically it is caused by the following: (i) in this regime, the quadrupole Galileon radiation dominates, which increases as $\propto P_{b}^{1 / 6}$ when $P_{b}$ becomes larger, and (ii) the quadrupole radiation in GR
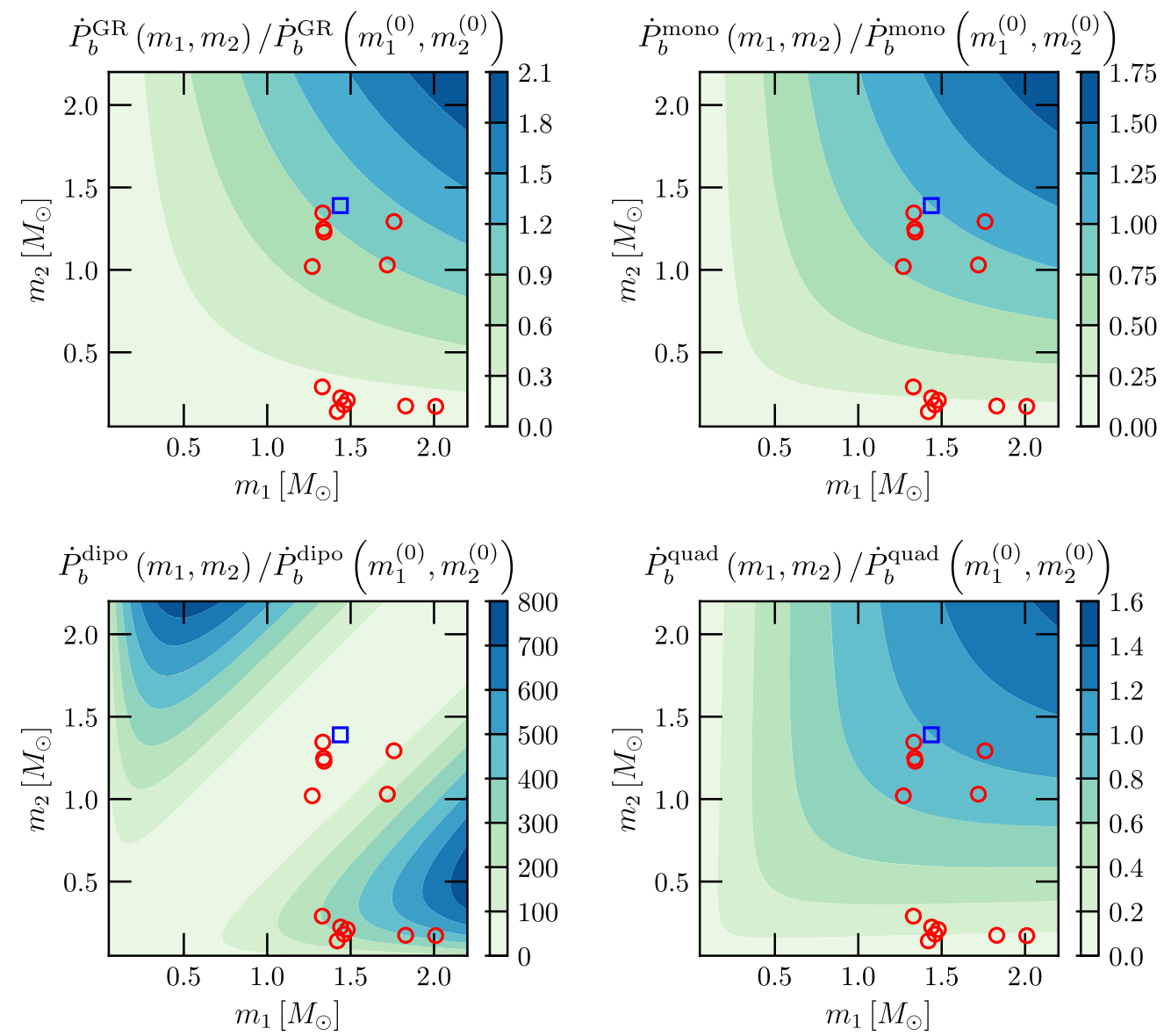

FIG. 5. The relative strength to $\dot{P}_{b}$ versus component masses $\left(m_{1}, m_{2}\right)$, with given orbital period $P_{b}$ and orbital eccentricity $e$. (Top left panel) The GR radiation. (Top right panel) monopole, (lower left panel) dipole, and (lower right) quadrupole Galileon radiation. The results are calculated with respect to a fiducial mass pair $m_{1}=1.438 M_{\odot}$ and $m_{2}=1.390 M_{\odot}$, denoted by the blue square. Red circles are the other 13 pulsars that are used in this paper (see Table I). 
decreases as $\propto P_{b}^{-5 / 3}$. With $m_{g}=10^{-27} \mathrm{eV} / c^{2}$ the Galileon radiation may even be larger than the GR one when $P_{b} \gtrsim$ day for nearly circular orbits. Moreover, we find that while the absolute Galileon quadrupole radiation increases with larger eccentricity (see Fig. 2), the relative contribution to GR decreases. This can be understood from the steepness of the curves in Fig. 2 for the quadrupole radiation in GR (black dots) and in the cubic Galileon (magenta circles). The latter is less steep, thus decreasing the relative ratio of $\dot{P}_{b}$ with larger eccentricity.

Lastly, to understand the effect of the component masses, in Fig. 5 we plot the relative strength to $\dot{P}_{b}$ for different mass pairs normalized to $\left(m_{1}, m_{2}\right)=$ $(1.438,1.390) M_{\odot}$, which is the fiducial mass pair based on the Hulse-Taylor pulsar [61]. Figure 5 is useful for analyzing binaries with a given orbital period and eccentricity but different component masses. It is well known that for the quadrupole radiation in GR, the so-called chirp mass, $\mathcal{M} \equiv\left(m_{1} m_{2}\right)^{3 / 5} / M^{1 / 5}$, plays a key role. Its contours in the $m_{1}-m_{2}$ parameter space are shown in the upper left panel in Fig. 5. The monopole and quadrupole Galileon radiations depend on the component masses in a quantitatively different—but qualitatively similar-way, as shown by the right panels in the figure. But for the dipole radiation, shown in the bottom left panel, the dependence is totally different. Asymmetric binaries are preferred for manifesting the dipole radiation. Notice that from the color bars, the enhancement from the asymmetric component masses for the dipole radiation can be as large as $\sim 10^{3}$, while for the other three types of radiation the change with component masses is within a relatively limited range.
This point will be clearly observed for pulsar-BH systems in Sec. IV.

\section{B. Constraints}

After having looked at the general features for the Galileon radiation in the last subsection, we now turn to realistic binary pulsar systems. We carefully choose 14 binary pulsars that are known to be precisely timed. They are chosen from the pool of millisecond pulsars in the second data release of the International Pulsar Timing Array program [66], as well as several other well-known systems with measurement of $\dot{P}_{b}[44,45,54,61,67-76]$. The relevant parameters for the test of the Galileon radiation are listed in Table I. Because binary pulsars have a large variety in terms of system parameters and observational characteristics, for detailed descriptions of these systems readers are referred to the original timing papers which are given for each pulsar in the table. As one can see from the last subsection, the Galileon radiation depends on a set of physical parameters of the binary pulsar system. These pulsars in Table I represent, to our best knowledge and resources, a set of the most suitable binary pulsar systems to date to perform the test in this paper.

For the chosen binary pulsars, post-Keplerian parameters other than $\dot{P}_{b}$ were used to calculate the component masses $m_{1}$ and $m_{2}$, assuming the validity of GR expressions $[12,78]$. The component masses are listed in the fourth and fifth columns in Table I. In the strictest sense, the component masses should be calculated consistently using cubic Galileon theory instead of GR. Nevertheless, as the Vainshtein suppression of the fifth force is more significant

TABLE I. Relevant parameters for a collection of binary pulsars for testing the Galileon radiation. Component masses were derived using post-Keplerian parameters other than $\dot{P}_{b}$, based on the validity of GR; $\sigma_{\dot{P}_{b}^{\text {obs }}}$ is the measurement uncertainty of the timing parameter $\dot{P}_{b}$, while $\sigma_{\dot{P}_{b} \text { int }}$ is the uncertainty of $\dot{P}_{b}$ after accounting for the kinematic Shklovskii effect and the galactic acceleration contribution $[77,78]$. Numbers in parentheses represent the $1 \sigma$ uncertainty in the last digit(s) quoted. For more details, interested readers are referred to the cited references alongside the pulsar name for each pulsar.

\begin{tabular}{|c|c|c|c|c|c|c|}
\hline Pulsar & $P_{b}(\mathrm{~d})$ & $e$ & $m_{1}\left(M_{\odot}\right)$ & $m_{2}\left(M_{\odot}\right)$ & $\sigma_{\dot{P}_{b}^{\mathrm{obs}}}\left(\mathrm{s} \mathrm{s}^{-1}\right)$ & $\sigma_{\dot{P}_{b}^{\text {int }}}\left(\mathrm{s} \mathrm{s}^{-1}\right)$ \\
\hline J0348 + 0432 [67] & $0.102424062722(7)$ & $(2.6 \pm 0.9) \times 10^{-6}$ & $2.01(4)$ & $0.172(3)$ & $4.5 \times 10^{-14}$ & $4.5 \times 10^{-14}$ \\
\hline J0437 - $4715[66,68]$ & $5.7410458(3)$ & $1.9182(1) \times 10^{-5}$ & $1.44(7)$ & $0.224(7)$ & $3 \times 10^{-15}$ & $2.8 \times 10^{-13}$ \\
\hline J0613 - $0200[66,69]$ & $1.198512575217(10)$ & $4.50(9) \times 10^{-6}$ & $1.42(46)$ & $0.14(3)$ & $7 \times 10^{-15}$ & $2.3 \times 10^{-14}$ \\
\hline J0737 - 3039A $[44,45]$ & $0.10225156248(5)$ & $0.0877775(9)$ & $1.3381(7)$ & $1.2489(7)$ & $1 \times 10^{-15}$ & $1 \times 10^{-15}$ \\
\hline $\mathrm{J} 1012+5307[66,70]$ & $0.604672723085(3)$ & $(1.1 \pm 0.1) \times 10^{-6}$ & $1.83(11)$ & $0.174(7)$ & $4 \times 10^{-15}$ & $8 \times 10^{-15}$ \\
\hline $\mathrm{J} 1022+1001[66,69]$ & $7.805136(1)$ & $9.704(5) \times 10^{-5}$ & $1.72(65)$ & $1.03(36)$ & $7 \times 10^{-14}$ & $2 \times 10^{-13}$ \\
\hline $\mathrm{J} 1141-6545[71,72]$ & $0.19765096149(3)$ & $0.171876(1)$ & $1.27(1)$ & $1.02(1)$ & $2.5 \times 10^{-14}$ & $2.5 \times 10^{-14}$ \\
\hline $\mathrm{B} 1534+12[73]$ & $0.420737298879(2)$ & $0.27367752(7)$ & $1.3330(2)$ & $1.3455(2)$ & $3 \times 10^{-16}$ & $1.1 \times 10^{-14}$ \\
\hline $\mathrm{J} 1713+0747[66,74]$ & $67.8251299228(5)$ & $7.49403(7) \times 10^{-5}$ & $1.33(10)$ & $0.290(11)$ & $1 \times 10^{-13}$ & $1 \times 10^{-13}$ \\
\hline $\mathrm{J} 1738+0333[54]$ & $0.3547907398724(13)$ & $(3.4 \pm 1.1) \times 10^{-7}$ & $1.46(6)$ & $0.181(8)$ & $3.1 \times 10^{-15}$ & $3.2 \times 10^{-15}$ \\
\hline $\mathrm{J} 1756$ - $2251[75]$ & $0.31963390143(3)$ & $0.1805694(2)$ & $1.341(7)$ & $1.230(7)$ & $5 \times 10^{-15}$ & $8 \times 10^{-15}$ \\
\hline J1909 - $3744[66,69]$ & $1.533449475278(1)$ & $1.04(6) \times 10^{-7}$ & $1.48(3)$ & $0.209(1)$ & $3 \times 10^{-15}$ & $1.4 \times 10^{-14}$ \\
\hline B1913 + $16[61]$ & $0.322997448918(3)$ & $0.6171340(4)$ & $1.438(1)$ & $1.390(1)$ & $1 \times 10^{-15}$ & $4 \times 10^{-15}$ \\
\hline $\mathrm{J} 2222-0137$ [76] & $2.44576456(13)$ & $0.380940(3) \times 10^{-4}$ & $1.76(6)$ & $1.293(25)$ & $9 \times 10^{-14}$ & $9 \times 10^{-14}$ \\
\hline
\end{tabular}



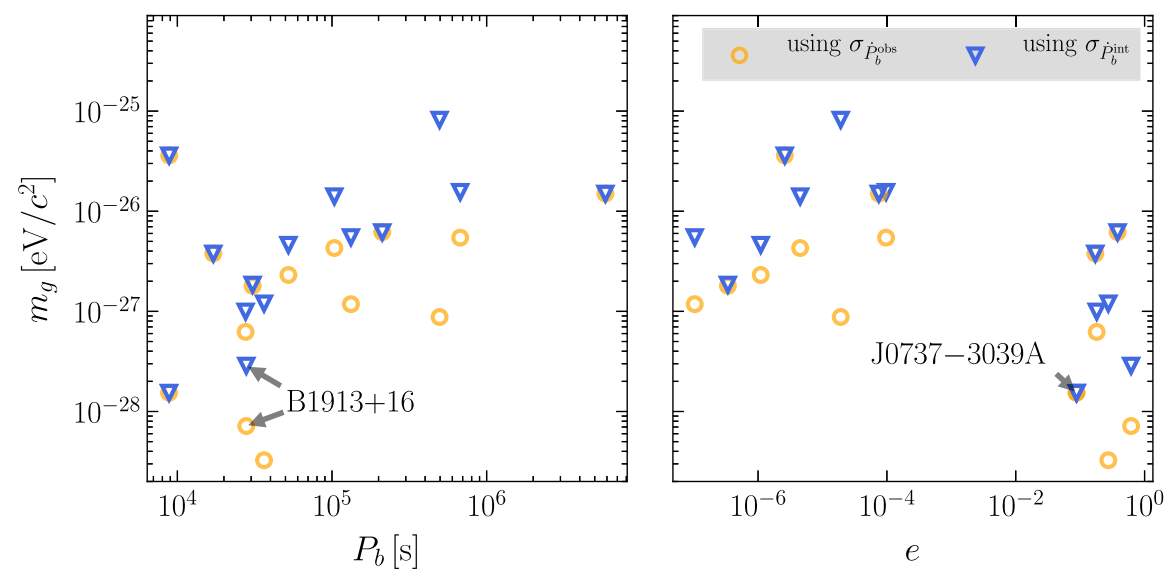

FIG. 6. Constraints on the graviton mass in cubic Galileon theory from individual binary pulsars versus their (left panel) orbital periods and (right panel) eccentricities at 68\% C.L. Orange circles and blue triangles are bounds obtained using $\sigma_{\dot{P}_{b}^{\text {obs }}}$ and $\sigma_{\dot{P}_{b}^{\text {int }}}$, respectively. The Hulse-Taylor pulsar PSR B1913 + 16 [61] and the double pulsar PSR J0737 - 3039A [45] are annotated.

than the Galileon radiation $[43,79]$, it is safe to use the GR formulas to extract these parameters.

As we can see in the penultimate column in the table, most of the chosen pulsars have uncertainties for the value of measured $\dot{P}_{b}$ at the level of $\sigma_{\dot{P}_{b}^{\text {obs }}} \sim 10^{-15} \mathrm{~s} \mathrm{~s}^{-1}$. PSR $\mathrm{B} 1534+12$ has an even better measurement, with $\sigma_{\dot{P}_{b}^{\text {obs }}} \simeq$ $3 \times 10^{-16} \mathrm{~s} \mathrm{~s}^{-1}$ [73]. The excellent timing precision is attributed to the long-term observation of these pulsars and the continuous improvements of the instruments at large radio telescopes $[78,80,81]$. However, these values cannot be directly used due to the astrophysical contribution and imperfect knowledge about their distances as well as the Milky Way's gravitational potential [77,78]. The most significant contribution comes from the kinematic Shklovskii effect and the galactic acceleration contribution. These contributions need to be subtracted using the measurement of the proper motion and the modeling of the galactic potential [77]. The subtraction introduces extra uncertainties in the intrinsic $\dot{P}_{b}$ parameter. The uncertainty after the subtraction, denoted as $\sigma_{\dot{P}_{b}^{\text {int }}}$, is listed in the last column of Table I for each pulsar.

All of the pulsars in the table have passed the tests of GR [12]. In particular, the measured orbital decay rates, after subtracting the kinematic Shklovskii effect and the galactic acceleration contribution, agree with the GR prediction in Eq. (9) within uncertainty for all binary pulsars that we consider. Therefore, here we do not look for evidence of the Galileon radiation; instead we put upper bounds on the graviton mass by constraining the extra Galileon radiation. By plainly assuming that the Galileon radiation is smaller than the uncertainty in $\dot{P}_{b}$, we obtain upper bounds for the graviton mass for each pulsar. These bounds are plotted in Fig. 6 for cases using $\sigma_{\dot{P}_{b}^{\text {int }}}$ and $\sigma_{\dot{P}_{b}^{\text {obs }}}$ as a function of the orbital period $P_{b}$ and the orbital eccentricity $e$.

The scenario of using $\sigma_{\dot{P}_{b}}$ should be taken as providing the current bounds on $m_{g}$. In this scenario, the best bound is given by the agreement of $\dot{P}_{b}$ with the GR prediction within $10^{-3}$ from the recent observation of the double pulsar PSR J0737 - 3039A [45]. The bound on $m_{g}$ reads

$$
m_{g} \lesssim 3 \times 10^{-28} \mathrm{eV} / c^{2} \quad(95 \% \text { C.L. }),
$$

which is 2 times better than the bound from the HulseTaylor pulsar PSR B1913+16, which gives $m_{g} \lesssim 6 \times$ $10^{-28} \mathrm{eV} / c^{2}$ (95\% C.L.) [61]. Although the larger eccentricity of PSR B1913 + $16(e \simeq 0.617)$ is beneficial for the test, compared with a mildly small eccentricity for PSR J0737 - 3039A ( $e \simeq 0.088$ ), the astrophysical contribution introduces a significant uncertainty to the intrinsic $\dot{P}_{b}$ of PSR B1913 + 16 [77]. The double pulsar, being relatively close to Earth, is not yet limited by the astrophysical contribution in $\dot{P}_{b}$ [44].

The scenario of using $\sigma_{\dot{P}_{b}^{\text {obs }}}$, on the other hand, should be taken as overly optimistic, representing the ability of clean binary pulsar systems in constraining the graviton mass. This is possible only when both the kinematic Shklovskii effect is measured and the galactic acceleration is modeled to be better than the observational uncertainty. In such an idealized case, the best pulsar would be PSR B1534 +12 [73], which gives $m_{g} \lesssim 6 \times 10^{-29} \mathrm{eV} / c^{2}$ (95\% C.L.) by itself alone. These overly optimistic results are also plotted in Fig. 6, with orange circles, but they are considered only to be indicative of technology capability and are not used in the following analysis.

Because the theory parameter, $m_{g}$, is universal in different pulsars, we can combine the constraints from individual pulsars into a single constraint. We assume that the measurements for different binary pulsars are independent; thus the covariant matrix is diagonal for them. We make use of a simple (logarithmic) likelihood $\ln \mathcal{L} \equiv$ $-\frac{1}{2} \sum_{i}\left(\dot{P}_{b}^{\pi} / \sigma_{\dot{P}_{b}^{\text {int }}}\right)^{2}$, where $\dot{P}_{b}^{\pi}$ is a sum of Galileon radiations in Eqs. (27)-(29), $\sigma_{\dot{P}_{b}^{\text {int }}}$ is as given in Table I, and the 


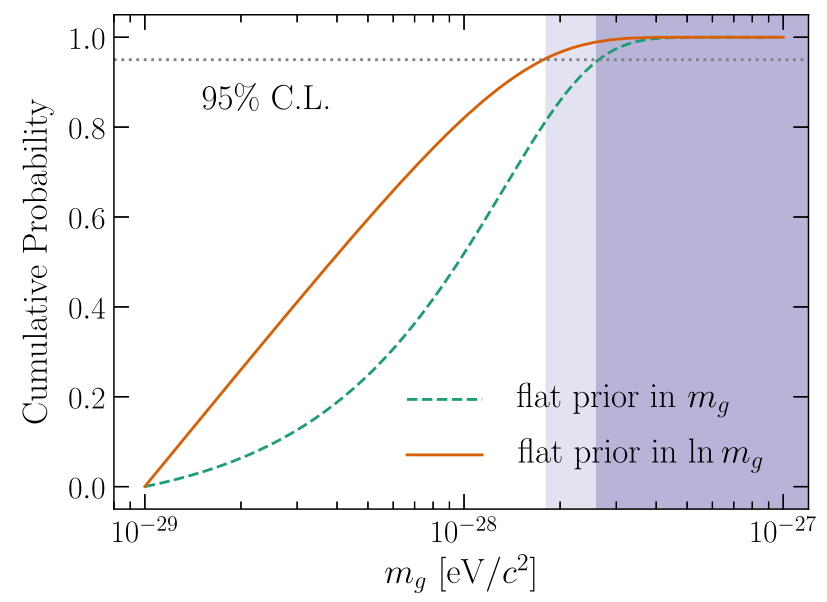

FIG. 7. Cumulative probability for the graviton mass with flat priors in $m_{g}$ (dashed line) and $\ln m_{g}$ (solid line). Shaded regions show the excluded graviton mass values at 95\% C.L. [see Eqs. (35) and (36)].

summation is over all binary pulsars considered in this paper. We can also assign prior knowledge to the graviton mass within the framework of Bayesian statistics [82]. In Fig. 7, we plot the combined cumulative probability distribution of the graviton mass for two different sets of prior knowledge. The combined bound is dominantly influenced by PSRs J0737-3039A and B1913+16, while the other 12 pulsars play only a minor role. From the figure, we obtain

$$
m_{g} \lesssim 3 \times 10^{-28} \mathrm{eV} / c^{2} \quad(95 \% \text { C.L. })
$$

when a uniform prior on $m_{g} \in\left(10^{-29}, 10^{-27}\right) \mathrm{eV} / c^{2}$ is assumed, and

$$
m_{g} \lesssim 2 \times 10^{-28} \mathrm{eV} / c^{2} \quad(95 \% \text { C.L. })
$$

when a uniform prior on $\ln m_{g}$ for $m_{g} \in\left(10^{-29}\right.$, $\left.10^{-27}\right) \mathrm{eV} / c^{2}$ is assumed; the high end of the prior range-namely, $10^{-27} \mathrm{eV} / \mathrm{c}^{2}$ - comes from the analysis in Ref. [43]. While the former bound [Eq. (35)] is very robust, the latter bound [Eq. (36)] using a uniform prior on $\ln m_{g}$ depends on our choice of prior range. It is a generic feature of Bayesian analysis. In a cosmologically favored reasoning, one might expect the graviton mass to be around the current Hubble scale-namely, $m_{g} \sim$ $H_{0} \sim 10^{-33} \mathrm{eV} / c^{2}$. If we had used a uniform prior on $\ln m_{g}$ for $m_{g} \in\left(10^{-34}, 10^{-27}\right) \mathrm{eV} / c^{2}$, we would have obtained a much tighter bound of $m_{g}$. However, such a bound is dominated by our prior knowledge. It means that binary pulsars are not yet sensitive to a cosmologically small graviton mass. Therefore, we stick to our relatively conservative result in Eq. (36). In contrast, the above change in the prior range does not affect the bound in Eq. (35) when a uniform prior on $m_{g}$ is adopted.
The results here for $m_{g}$ improve on those in Ref. [43], $m_{g} \lesssim 10^{-27} \mathrm{eV} / c^{2}$ (95\% C.L.) due to uses of an updated analysis and recent observational results of pulsar timing, in particular, use of the double pulsar results given in Ref. [45].

\section{PROJECTED CONSTRAINTS WITH PULSAR-BH SYSTEMS}

A well-timed pulsar around a BH companion is a longsought-after holy grail in pulsar astronomy. The discovery of these systems will enable a couple of unprecedented tests of gravity theories, in particular, on the aspects related to the property of $\mathrm{BH}$ solutions $[17,46,48,80,81,83]$. To date, despite extensive dedicated searches, no conclusively convincing candidate has been found. The uncertainty in the estimation of the number of these potential systems pertains mainly to their formation channels. We will not further discuss the involved astrophysics here. Nevertheless, several studies have shown good potential for discovering pulsar-BH systems in the near future $[47,49,51,52,84]$. If such a pulsar-BH system is discovered, it will provide a completely new playground in which to perform interesting tests of gravity, including Galileon radiation [48].

In Fig. 8, we plot the expected contribution of Galileon radiation to the orbital decay rate for a pulsar- $10 M_{\odot} \mathrm{BH}$ system. In the figure, the graviton mass is assumed to be $m_{g}=10^{-28} \mathrm{eV} / c^{2}$, which is roughly the best bound from the combination of the current binary pulsars, obtained in Eqs. (35) and (36). Because of the asymmetric masses, for extreme (unrealistic) systems with $P_{b} \lesssim 1 \mathrm{~min}$ and $e \gtrsim 0.95$, the Galileon dipole radiation could be relevant to the total radiation. However, even if any such systems exist, they are unlikely to be detected with radio telescopes due to the large orbital acceleration and limited computation resources [47]; however, fast imaging and imaging searches based on significant circular polarization or scintillation might help [85-87]. The expected number for the LISA detector is very low as well due to their short lifetimes before the merger [64]. For binaries with $P_{b} \gtrsim 1$ day, the Galileon quadrupole radiation is still the dominant contributor.

Depending on the eccentricity, the Galileon monopole radiation could now play an essential role for binaries with $P_{b} \lesssim 1$ day. Discovery of such binaries might be realistic for current and upcoming radio telescopes [47,80]. Liu et al. [47] conducted extensive time-of-arrival (TOA) simulation for a pulsar-BH system with masses $\left(m_{1}, m_{2}\right)=$ $(1.4,10) M_{\odot}$.

As with Ref. [47], we have conducted extensive mockdata simulations for different pulsar-BH configurations. For all of these simulations, we have assumed one observing session per week with ten TOAs of given uncertainty $\sigma_{\mathrm{TOA}}$ over a period of five years. We further assume that the TOAs follow a Gaussian distribution and are uncorrelated 


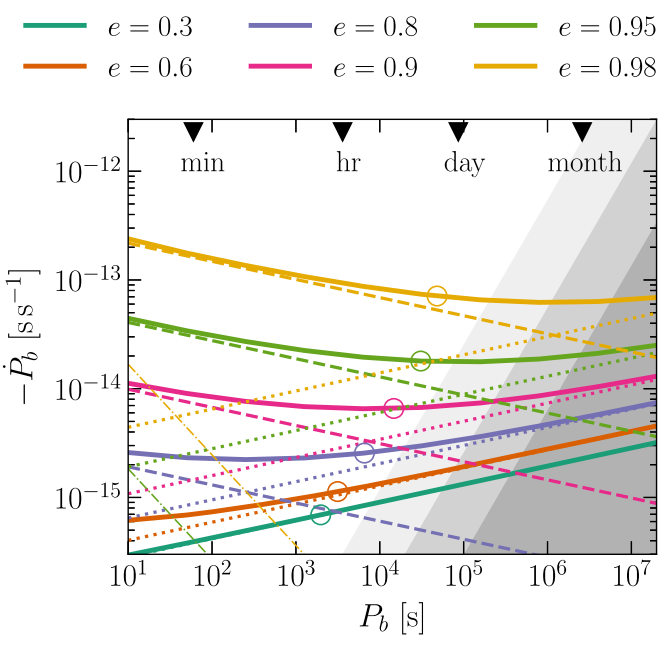

FIG. 8. Same as Fig. 3, but for $m_{1}=1.4 M_{\odot}, m_{2}=10 M_{\odot}$, and $m_{g}=10^{-28} \mathrm{eV} / c^{2}$. Open circles represent systems that have lifetime of $10^{6} \mathrm{yr}$ before the merger in GR. Shaded regions show the estimated measurement precision that can be achieved with (from dark to light) $\sigma_{\mathrm{TOA}}=0.1,1$, and $10 \mu \mathrm{s}$; the precision is found to be independent of eccentricity. Note that our simulations are performed for orbits with $P_{b} \gtrsim 0.2$ day, and in this figure orbits with $P_{b} \lesssim 0.2$ day are extrapolated using Eq. (37).

(white noise). Our simulations cover an orbital period range from 0.2 to about 100 days. We find that with a given orbital period the precision in measuring $\dot{P}_{b}$, denoted by the (dimensionless) quantity $\sigma_{\dot{P}_{b}}$, is only weakly dependent on the orbital eccentricity. The dependence on $P_{b}$ is nicely fitted by

$$
\log _{10} \sigma_{\dot{P}_{b}}=\mathcal{A}+\mathcal{B} \log _{10}\left(\frac{P_{b}}{\text { day }}\right)
$$

where $(\mathcal{A}, \mathcal{B})=(-15.67,1.331),(-14.67,1.331)$, and $(-13.68,1.332)$, when $\sigma_{\mathrm{TOA}}$ is $0.1,1$, and $10 \mu \mathrm{s}$, respectively. In fact, to good approximation one can use $\mathcal{A}=$ $-14.7+\log _{10}\left(\sigma_{\mathrm{TOA}} / \mu \mathrm{s}\right)$ and $\mathcal{B}=1.33$. The actual uncertainty obtained for a TOA depends on various aspects, like pulsar luminosity, pulse profile, integration time, and telescope and backend parameters. The assumed uncertainties and number of TOAs are typical for precision timing observations in pulsar astronomy (see, e.g., Ref. [66]). The expected precision for the three different values of $\sigma_{\mathrm{TOA}}$ is shown as shaded regions in Fig. 8. We see that pulsar-BH systems have great potential to improve the current best bound on $m_{g}$. For example, if a $\sigma_{\mathrm{TOA}}$ of about $0.1 \mu \mathrm{s}(10 \mu \mathrm{s})$ is achieved, an eccentric pulsar with $P_{b} \lesssim 1$ month $\left(P_{b} \lesssim 1\right.$ day) can probe $m_{g}$ down to the level of $10^{-28} \mathrm{eV} / c^{2}$. We emphasize that the timing precision assumed here can generally be achieved only for recycled pulsars, even with large radio telescopes like the Fivehundred-meter Aperture Spherical Telescope [88,89] or the upcoming Square Kilometre Array $[80,81,83]$. Such pulsar-BH systems could, in principle, be the result of an

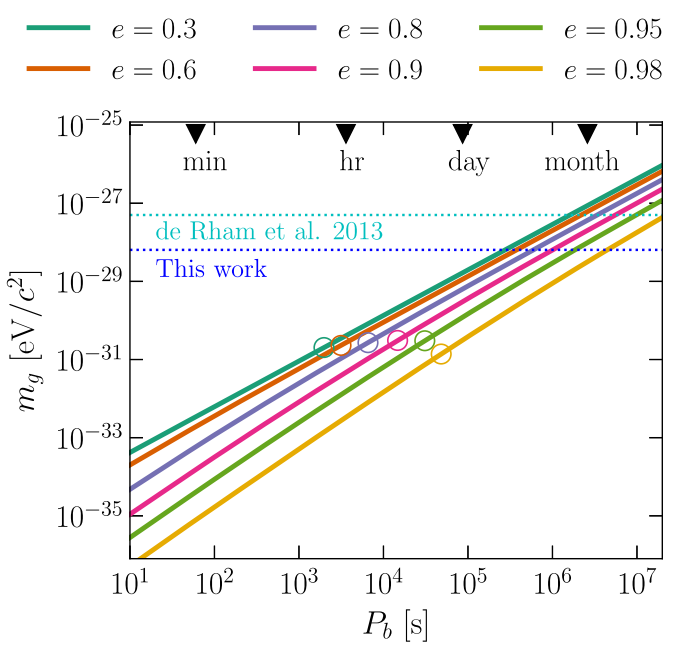

FIG. 9. Projected bounds at $68 \%$ C.L. on $m_{g}$ from a pulsar-BH system with $\sigma_{\mathrm{TOA}}=0.1 \mu \mathrm{s}$. Open circles represent systems that have a lifetime of $10^{6} \mathrm{yr}$ before merger in GR. As in Fig. 8, results for orbits with $P_{b} \lesssim 0.2$ day are extrapolated using Eq. (37).

exchange encounter in regions with high stellar density, like globular clusters and the Galactic Center region (see, e.g., Ref. [90]). The actual timing precision will, in addition, depend on other parameters like pulsar luminosity and pulse shape.

In Fig. 9, we plot the projected bounds on $m_{g}$ using the $\dot{P}_{b}$ precision in Eq. (37) with $\sigma_{\mathrm{TOA}}=0.1 \mu \mathrm{s}$. As we can see in the figure, if we can time a near-circular pulsar-BH binary with $P_{b}$ smaller than a few days, the bound [Eq. (36)] in this paper can be improved. If the binary is highly eccentric, then an orbital period $P_{b}$ smaller than a few months is sufficient to improve the bound, as was indicated in Fig. 8. On the other hand, if one wants to probe the cosmologically interested range for $m_{g} \sim 10^{-33} \mathrm{eV} / c^{2}$ [43], a subminute circular binary or a subhour highly eccentric binary is needed. We consider such cases highly unlikely to be discovered with near-future technologies, leaving aside the fact that the existence of such a system in our Galaxy is almost certainly excluded due to its short merger time.

In another direction, extensive searches for pulsars around the $\mathrm{Sgr} \mathrm{A}^{*}$, the supermassive $\mathrm{BH}$ at the center of our Galaxy, are ongoing [49,51,52,91]. In Fig. 10, we plot the Galileon radiation for a pulsar-Sgr A* $\mathrm{BH}$ system for different eccentricities with $m_{g}=10^{-28} \mathrm{eV} / c^{2}$. For eccentric systems with $P_{b} \lesssim 1 \mathrm{yr}$, the Galileon monopole radiation prevails over the Galileon quadrupole radiation for $e \gtrsim 0.6$. Therefore, there might be an opportunity to test the Galileon monopole radiation within this class of systems. To address this question, we perform mock-data simulations to investigate the precision one can expect for $\dot{P}_{b}$ for a pulsar in a suitable orbit around $\mathrm{Sgr} \mathrm{A}^{*}$. As was done in Ref. [49], we have created mock data with one TOA of 


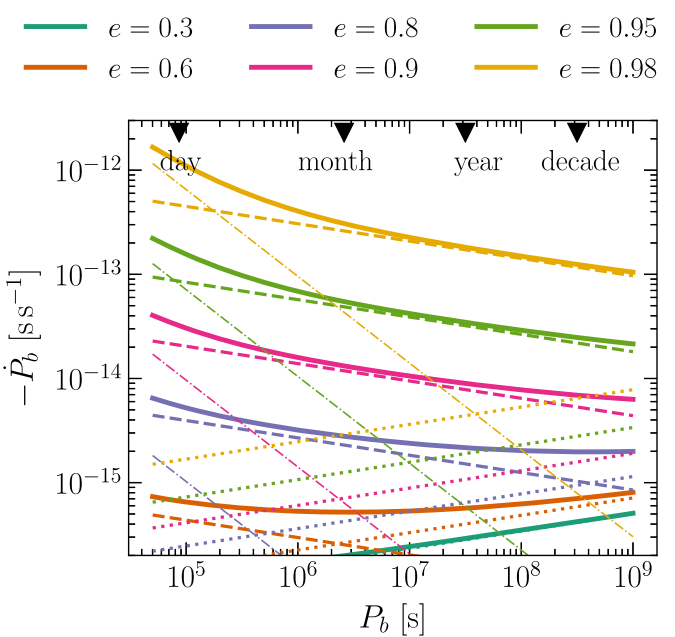

FIG. 10. Same as Fig. 3, but for $m_{1}=1.4 M_{\odot}, m_{2}=$ $4 \times 10^{6} M_{\odot}$, and $m_{g}=10^{-28} \mathrm{eV} / c^{2}$.

$100 \mu$ s precision every week over a time span of five years. Furthermore, we have assumed that the pulsar orbit is unperturbed, and therefore our parameter estimation is based on a phase-connected timing solution that provides a perfect fit over the whole time span of observations. Even under such optimistic assumptions, we find, for an orbit with $P_{b}=0.5 \mathrm{yr}$ and $e=0.8$, that it is unlikely to get a $\sigma_{\dot{P}_{b}}$ better than $10^{-12} \mathrm{~s} \mathrm{~s}^{-1}$. Therefore, only in the event of a pulsar in a highly eccentric orbit with $P_{b} \lesssim 1$ day being discovered might we be able to improve the bound [Eq. (36)]. However, we consider the existence of a pulsar in such an orbit extremely unlikely.

The tests in this section depend sensitively on the actual eccentricity of the pulsar-BH system, as shown in Figs. 8-10. In fact, the analysis with Figs. 8 and 10 is conservative because the Galileon radiation power was calculated by averaging over the orbital timescale [43]. In reality, during the periastron passage the gravitational radiation is maximized and produces prominent features. These features are believed to provide even more distinguishable signals. An analysis resolving the orbital timescale is beyond the scope of this paper. Because all three kinds of Galileon radiation are proportional to $m_{g}$, the results discussed in Figs. 8 and 10 can be rescaled easily with different graviton masses for different binary systems.

\section{DISCUSSION}

In this paper, we systematically studied the Galileon radiation in cubic Galileon theory [43] in the context of pulsar timing. Because the Galileon radiation is screened differently than its fifth-force counterpart, such a study is essential to better understand the basic role of the Vainshtein mechanism in screening the gravitational radiation.
From a collection of 14 well-timed binary pulsars, we obtained a new bound on the theory parameter for the cubic Galileon, namely, the graviton mass

$$
m_{g} \lesssim 2 \times 10^{-28} \mathrm{eV} / c^{2} \quad(95 \% \text { C.L. })
$$

when a uniform prior on $\ln m_{g}$ for $m_{g} \in$ $\left(10^{-29}, 10^{-27}\right) \mathrm{eV} / \mathrm{c}^{2}$ is used. This improves on a previous bound from the Hulse-Taylor pulsar [43] by a factor of 5 . Though the bound [Eq. (38)] is weaker than a few other bounds, such as the bounds from the Earth-Moon-Sun system and dark matter clusters [23], it is nevertheless a robust bound from a completely different regime-namely, from the dynamic gravitational radiation instead of the static environments. It is also immune from uncertain assumptions about the dark matter distributions and the virialization of the gravitating systems. Therefore, we consider this bound complementary to the existing bounds.

Finally, de Rham et al. [79] discussed radiation from a generic Galileon theory with all allowed interactions in four-dimensional spacetime. The inclusion of quartic or quintic Galileon complicates the calculations considerably. The authors found that naive perturbation theory predicts divergent results in the radiation power when these higherorder terms are considered, meaning that the perturbations themselves are nonlinear. Partial results were obtained for binary systems with specific assumptions about the screening length scales. In particular, only circular binary orbits were analyzed, and they are not applicable to most of the systems that we considered in this paper. For circular orbits that were considered in Ref. [79], meaningful bounds can be derived when there is a hierarchy of strong coupling scales. We wish to perform a more complete analysis with higher-order Galileon interactions in a future study.

\section{ACKNOWLEDGMENTS}

We are grateful to Paulo Freire, Xueli Miao, Robert Wharton, and Kent Yagi for the helpful discussions, and to Vivek Venkatraman Krishnan for carefully reading the manuscript. This work was supported by the National Natural Science Foundation of China (Grants No. 11975027, No. 11991053, and No. 11721303), the Young Elite Scientists Sponsorship Program by the China Association for Science and Technology (Grant No. 2018QNRC001), the Max Planck Partner Group Program, funded by the Max Planck Society, and the High-Performance Computing Platform of Peking University. L. S. and N. W. acknowledge support from the European Research Council (ERC) for the ERC Synergy Grant BlackHoleCam under Contract No. 610058. S.-Y. Z. acknowledges support from the starting grants from University of Science and Technology of China under Grants No. KY2030000089 and No. GG2030040375 and is also supported by the National Natural Science Foundation of China (NSFC) under Grant No. 11947301. 
[1] M. Li, X.-D. Li, S. Wang, and Y. Wang, Commun. Theor. Phys. 56, 525 (2011).

[2] J. Gleyzes, D. Langlois, F. Piazza, and F. Vernizzi, J. Cosmol. Astropart. Phys. 08 (2013) 025.

[3] A. Joyce, B. Jain, J. Khoury, and M. Trodden, Phys. Rep. 568, 1 (2015).

[4] S. Weinberg, Rev. Mod. Phys. 61, 1 (1989).

[5] N. Aghanim et al. (Planck Collaboration), arXiv:1807 .06209 .

[6] S. Birrer et al., Mon. Not. R. Astron. Soc. 484, 4726 (2019).

[7] A. G. Riess, S. Casertano, W. Yuan, L. M. Macri, and D. Scolnic, Astrophys. J. 876, 85 (2019).

[8] T. Clifton, P. G. Ferreira, A. Padilla, and C. Skordis, Phys. Rep. 513, 1 (2012).

[9] E. Berti et al., Classical Quantum Gravity 32, 243001 (2015).

[10] L. Heisenberg, Phys. Rep. 796, 1 (2019).

[11] C. M. Will, Living Rev. Relativity 17, 4 (2014).

[12] N. Wex, in Frontiers in Relativistic Celestial Mechanics: Applications and Experiments, Vol. 2, edited by S.M. Kopeikin (Walter de Gruyter GmbH, Berlin, 2014), p. 39.

[13] L. Shao and N. Wex, Sci. China Phys. Mech. Astron. 59, 699501 (2016).

[14] N. Yunes and S. A. Hughes, Phys. Rev. D 82, 082002 (2010).

[15] K. Yagi, D. Blas, N. Yunes, and E. Barausse, Phys. Rev. Lett. 112, 161101 (2014).

[16] K. Yagi, D. Blas, E. Barausse, and N. Yunes, Phys. Rev. D 89, 084067 (2014).

[17] L. Shao, N. Wex, and M. Kramer, Phys. Rev. Lett. 120, 241104 (2018).

[18] M. A. Luty, M. Porrati, and R. Rattazzi, J. High Energy Phys. 09 (2003) 029.

[19] A. Nicolis, R. Rattazzi, and E. Trincherini, Phys. Rev. D 79, 064036 (2009).

[20] A. I. Vainshtein, Phys. Lett. 39B, 393 (1972).

[21] E. Babichev and C. Deffayet, Classical Quantum Gravity 30, 184001 (2013).

[22] C. de Rham, Living Rev. Relativity 17, 7 (2014).

[23] C. de Rham, J. T. Deskins, A. J. Tolley, and S.-Y. Zhou, Rev. Mod. Phys. 89, 025004 (2017).

[24] H. van Dam and M. J. G. Veltman, Nucl. Phys. B22, 397 (1970).

[25] V. I. Zakharov, JETP Lett. 12, 312 (1970), https://ui.adsabs .harvard.edu/abs/1970JETPL..12..312Z.

[26] Y. Iwasaki, Phys. Rev. D 2, 2255 (1970).

[27] C. de Rham, G. Gabadadze, and A. J. Tolley, Phys. Rev. Lett. 106, 231101 (2011).

[28] C. de Rham and G. Gabadadze, Phys. Rev. D 82, 044020 (2010).

[29] G. R. Dvali, G. Gabadadze, and M. Porrati, Phys. Lett. B 484, 112 (2000).

[30] S. F. Hassan and R. A. Rosen, J. High Energy Phys. 02 (2012) 126.

[31] A. Padilla, P. M. Saffin, and S.-Y. Zhou, J. High Energy Phys. 12 (2010) 031.

[32] A. Padilla, P. M. Saffin, and S.-Y. Zhou, J. High Energy Phys. 01 (2011) 099.

[33] A. Padilla, P. M. Saffin, and S.-Y. Zhou, Phys. Rev. D 83, 045009 (2011).

[34] G. W. Horndeski, Int. J. Theor. Phys. 10, 363 (1974).
[35] C. Deffayet, X. Gao, D. A. Steer, and G. Zahariade, Phys. Rev. D 84, 064039 (2011).

[36] B. P. Abbott et al., Phys. Rev. Lett. 119, 161101 (2017).

[37] B. P. Abbott et al., Astrophys. J. 848, L13 (2017).

[38] T. Baker, E. Bellini, P. G. Ferreira, M. Lagos, J. Noller, and I. Sawicki, Phys. Rev. Lett. 119, 251301 (2017).

[39] J. M. Ezquiaga and M. Zumalacárregui, Phys. Rev. Lett. 119, 251304 (2017).

[40] P. Creminelli and F. Vernizzi, Phys. Rev. Lett. 119, 251302 (2017).

[41] C. de Rham and S. Melville, Phys. Rev. Lett. 121, 221101 (2018).

[42] G. Dvali, A. Gruzinov, and M. Zaldarriaga, Phys. Rev. D 68, 024012 (2003).

[43] C. de Rham, A. J. Tolley, and D. H. Wesley, Phys. Rev. D 87, 044025 (2013).

[44] M. Kramer et al., Science 314, 97 (2006).

[45] M. Kramer, Int. J. Mod. Phys. D 25, 1630029 (2016).

[46] N. Wex and S. Kopeikin, Astrophys. J. 514, 388 (1999).

[47] K. Liu, R. P. Eatough, N. Wex, and M. Kramer, Mon. Not. R. Astron. Soc. 445, 3115 (2014).

[48] B. C. Seymour and K. Yagi, Phys. Rev. D 98, 124007 (2018).

[49] K. Liu, N. Wex, M. Kramer, J. M. Cordes, and T. J. W. Lazio, Astrophys. J. 747, 1 (2012).

[50] D. Psaltis, N. Wex, and M. Kramer, Astrophys. J. 818, 121 (2016).

[51] G. C. Bower et al., ASP Conf. Ser. 517, 793 (2018), https:// ui.adsabs.harvard.edu/abs/2018ASPC..517..793B.

[52] G. C. Bower et al., Bull. Am. Astron. Soc. 51, 438 (2019), https://ui.adsabs.harvard.edu/abs/2019BAAS...51c.438B.

[53] J. H. Taylor, L. A. Fowler, and P. M. McCulloch, Nature (London) 277, 437 (1979).

[54] P. C. C. Freire, N. Wex, G. Esposito-Farèse, J. P. W. Verbiest, M. Bailes, B. A. Jacoby, M. Kramer, I. H. Stairs, J. Antoniadis, and G. H. Janssen, Mon. Not. R. Astron. Soc. 423, 3328 (2012).

[55] K. Yagi, L. C. Stein, and N. Yunes, Phys. Rev. D 93, 024010 (2016).

[56] L. Shao, N. Sennett, A. Buonanno, M. Kramer, and N. Wex, Phys. Rev. X 7, 041025 (2017).

[57] J. Zhao, L. Shao, Z. Cao, and B.-Q. Ma, Phys. Rev. D 100, 064034 (2019).

[58] P. C. Peters and J. Mathews, Phys. Rev. 131, 435 (1963).

[59] Y.-Z. Chu and M. Trodden, Phys. Rev. D 87, 024011 (2013).

[60] F. Dar, C. De Rham, J. T. Deskins, J. T. Giblin, and A. J. Tolley, Classical Quantum Gravity 36, 025008 (2019).

[61] J. M. Weisberg and Y. Huang, Astrophys. J. 829, 55 (2016).

[62] L. S. Finn and P. J. Sutton, Phys. Rev. D 65, 044022 (2002).

[63] X. Miao, L. Shao, and B.-Q. Ma, Phys. Rev. D 99, 123015 (2019).

[64] P. Amaro-Seoane et al. (LISA Collaboration), arXiv:1702 .00786

[65] E. Barausse et al., Gen. Relativ. Gravit. (to be published).

[66] B. B. P. Perera et al., Mon. Not. R. Astron. Soc. 490, 4666 (2019).

[67] J. Antoniadis et al., Science 340, 1233232 (2013).

[68] D. J. Reardon et al., Mon. Not. R. Astron. Soc. 455, 1751 (2016).

[69] G. Desvignes et al., Mon. Not. R. Astron. Soc. 458, 3341 (2016). 
[70] K. Lazaridis et al., Mon. Not. R. Astron. Soc. 400, 805 (2009).

[71] N. D. R. Bhat, M. Bailes, and J. P. W. Verbiest, Phys. Rev. D 77, 124017 (2008).

[72] V. V. Krishnan et al., Science 367, 577 (2020).

[73] E. Fonseca, I. H. Stairs, and S. E. Thorsett, Astrophys. J. 787, 82 (2014).

[74] W. W. Zhu et al., Mon. Not. R. Astron. Soc. 482, 3249 (2019).

[75] R. D. Ferdman et al., Mon. Not. R. Astron. Soc. 443, 2183 (2014).

[76] I. Cognard et al., Astrophys. J. 844, 128 (2017).

[77] T. Damour and J. H. Taylor, Astrophys. J. 366, 501 (1991).

[78] D. R. Lorimer and M. Kramer, Handbook of Pulsar Astronomy (Cambridge University Press, Cambridge, England, 2005).

[79] C. de Rham, A. Matas, and A. J. Tolley, Phys. Rev. D 87, 064024 (2013).

[80] M. Kramer, D. C. Backer, J. M. Cordes, T. J. W. Lazio, B. W. Stappers, and S. Johnston, New Astron. Rev. 48, 993 (2004).

[81] L. Shao et al., Proc. Sci., AASKA14 (2015) 042.
[82] W. Del Pozzo and A. Vecchio, Mon. Not. R. Astron. Soc. 462, L21 (2016).

[83] A. Weltman et al., Pub. Astron. Soc. Aust. 37, e002 (2020).

[84] R. S. Wharton, S. Chatterjee, J. M. Cordes, J. S. Deneva, and T. J. W. Lazio, Astrophys. J. 753, 108 (2012).

[85] C. J. Law, G. C. Bower, S. Burke-Spolaor, B. J. Butler, P. Demorest, A. Halle, S. Khudikyan, T. J. W. Lazio, M. Pokorny, J. Robnett, and M. P. Rupen, Astrophys. J. Suppl. Ser. 236, 8 (2018).

[86] D. L. Kaplan et al., Astrophys. J. 884, 96 (2019).

[87] S. Dai, S. Johnston, M.E. Bell, W. A. Coles, G. Hobbs, R. D. Ekers, and E. Lenc, Mon. Not. R. Astron. Soc. 462, 3115 (2016).

[88] P. Jiang, Y.-L. Yue, H.-Q. Gan, R. Yao, H. Li, G.-F. Pan, J.-H. Sun, D.-J. Yu, H.-F. Liu, N.-Y. Tang, L. Qian, J.-G. Lu, J. Yan, B. Peng, S.-X. Zhang, Q.-M. Wang, Q. Li, and D. Li, Sci. China Phys. Mech. Astron. 62, 959502 (2019).

[89] J.-G. Lu, K.-J. Lee, and R.-X. Xu, Sci. China Phys. Mech. Astron. 63, 229531 (2019).

[90] C.-A. Faucher-Gigure and A. Loeb, Mon. Not. R. Astron. Soc. 415, 3951 (2011).

[91] C. Goddi et al., Int. J. Mod. Phys. D 26, 1730001 (2016). 